University of Nebraska - Lincoln

DigitalCommons@University of Nebraska - Lincoln

\title{
Absolute Doubly Differential Cross Sections for Ejection of Secondary Electrons from Gases by Electron Impact. II. 100-500-eV Electrons on Neon, Argon, Molecular Hydrogen, and Molecular Nitrogen
}

R. D. DuBois

University of Nebraska - Lincoln

M. Eugene Rudd

University of Nebraska - Lincoln, erudd@unl.edu

Follow this and additional works at: https://digitalcommons.unl.edu/physicsrudd

Part of the Physics Commons

DuBois, R. D. and Rudd, M. Eugene, "Absolute Doubly Differential Cross Sections for Ejection of Secondary Electrons from Gases by Electron Impact. II. 100-500-eV Electrons on Neon, Argon, Molecular Hydrogen, and Molecular Nitrogen" (1978). M. Eugene Rudd Publications. 47.

https://digitalcommons.unl.edu/physicsrudd/47

This Article is brought to you for free and open access by the Research Papers in Physics and Astronomy at DigitalCommons@University of Nebraska - Lincoln. It has been accepted for inclusion in M. Eugene Rudd Publications by an authorized administrator of DigitalCommons@University of Nebraska - Lincoln. 


\title{
Physical Review A 17, 843 - 848 (1978)
}

\section{Absolute doubly differential cross sections for ejection of secondary electrons from gases by electron impact. II. 100-500-eV electrons on neon, argon, molecular hydrogen, and molecular nitrogen}

\author{
R. D. DuBois * and M. E. Rudd
}

Behlen Laboratory of Physics, University of Nebraska, Lincoln, Nebraska 68588

Received 22 September 1977

Absolute doubly differential cross sections for secondary electron production by electron impact have been measured for static gas targets of neon, argon, hydrogen, and nitrogen. Electron impact energies were from 100 to $500 \mathrm{eV}$. An electrostatic analyzer was used to analyze secondary electrons with energies between $4 \mathrm{eV}$ and the primary electron energy minus the first ionization potential of the target. Angles of emission were $10^{\circ}$ to $150^{\circ}$. The present data agree well with the data of Opal, Beaty, and Peterson at $90^{\circ}$ but, as was observed previously for helium, the agreement becomes increasingly poorer for larger and smaller angles. This angular disagreement, which is independent of target gas and impact energy, is approximately given by $a+(1-a) \sin \theta$, where $a=0.10 \pm 0.12$. Previously we compared the experimental data of Opal, Beaty, and Peterson with calculations by Manson for helium and obtained a similar correction but with $a=0.53$. Recent Born-approximation calculations of Manson are compared with our 500$\mathrm{eV}$ argon data. The calculations reproduce the angular distributions of the measured cross sections quite well for small secondary-electron energies. For intermediate energies the agreement is still quite good near the momentum-conservation peak but poorer for large scattering angles.

(C)1978 The American Physical Society

URL: http://link.aps.org/doi/10.1103/PhysRevA.17.843

DOI: $10.1103 /$ PhysRevA.17.843

* Present address: Institute of Physics, University of Aarhus, DK 8000 Aarhus C, Denmark. 


\title{
Absolute doubly differential cross sections for ejection of secondary electrons from gases by electron impact. II. 100-500-eV electrons on neon, argon, molecular hydrogen, and molecular nitrogen
}

\author{
R. D. DuBois* and M. E. Rudd \\ Behlen Laboratory of Physics, University of Nebraska, Lincoln, Nebraska 68588
}

(Received 22 September 1977)

\begin{abstract}
Absolute doubly differential cross sections for secondary electron production by electron impact have been measured for static gas targets of neon, argon, hydrogen, and nitrogen. Electron impact energies were from 100 to $500 \mathrm{eV}$. An electrostatic analyzer was used to analyze secondary electrons with energies between $4 \mathrm{eV}$ and the primary electron energy minus the first ionization potential of the target. Angles of emission were $10^{\circ}$ to $150^{\circ}$. The present data agree well with the data of Opal, Beaty, and Peterson at $90^{\circ}$ but, as was observed previously for helium, the agreement becomes increasingly poorer for larger and smaller angles. This angular disagreement, which is independent of target gas and impact energy, is approximately given by $a+(1-a) \sin \theta$, where $a=0.10 \pm 0.12$. Previously we compared the experimental data of Opal, Beaty, and Peterson with calculations by Manson for helium and obtained a similar correction but with $a=0.53$. Recent Born-approximation calculations of Manson are compared with our 500-eV argon data. The calculations reproduce the angular distributions of the measured cross sections quite well for small secondary, electron energies. For intermediate energies the agreement is still quite good near the momentumconservation peak but poorer for large scattering angles.
\end{abstract}

\section{INTRODUCTION}

When an energetic electron passes through a gas, ionizing collisions will occur. Such a collision results in a scattered incident electron with degraded energy, an ejected atomic electron, and a recoiling ion. Detailed information about these collisions may be given in terms of the doubly differential cross sections (DDCS) for ejection of secondary electrons of energy $E_{s}$ (this also includes scattered electrons) at an angle $\theta$ by a primary electron of energy $E_{p}$. The DDCS are also written $\sigma\left(E_{p}, E_{s}, \theta\right)$ or $\sigma\left(E_{s}, \theta\right)$. These cross sections are of interest in plasma, atmospheric, and radiation physics, as well as having intrinsic value because of their basic nature. Since the collision is a three-body process in which exchange is possible, it is difficult to treat theoretically.

Previous experimental measurements of DDCS by electron impact for targets other than helium are few. The most extensive study is that of Opal, Beaty, and Peterson, ${ }^{1}$ henceforth to be called OBP. They present data for several gases and a large range of primary energies in tabular form but their secondary electron energy range does not include the higher energies where most of the scattered primary electrons of degraded energy are found. Oda ${ }^{2}$ has measured cross sections over the entire ionization continuum for several targets using $500 \mathrm{eV}$ primary electrons. More recent are the data reported by Kennerly and Bonham $^{3}$ employing the time-of-flight technique originally used by Toburen and Wilson ${ }^{4}$ for proton impact. Also noted are the data of Tisone. ${ }^{5}$ These data are thus far for limited angular or energy ranges and few gases.

Previously (paper I) we presented measurements of DDCS for helium. ${ }^{6}$ A comparison of our static gas measurements with the directed beam work of OBP indicated that an angular correction needs to be applied to their data. Instead of using our data to determine the correction, however, we made an extensive comparison of OBP's data with the Born approximation calculations of Manson et $a l .{ }^{7}$ at $2 \mathrm{keV}$ and found that the correction needed to bring OBP's data into approximate agreement was identical with the correction suggested by an auxiliary experiment reported by OBP themselves. This correction was to divide the data of OBP by the factor $a+(1-a) \sin \theta$ with $a=0.53$. Comparisons made with our experimental data on helium and with that of earlier investigators indicated the need for a stronger correction, i.e., a smaller value of the constant $a$. However, at that time comparisons had only been made at $30^{\circ}$ and $90^{\circ}$. We now have comparisons at all angles and for several additional gases. Because our measurements are made with static gas targets the calculation of the interaction volume is simple and straightforward using only the standard $1 / \sin \theta$ factor. Because of our good angular resolution, this is a good approximation even down to angles as small as $2^{\circ}$ as shown in our earlier work on elastic scattering. ${ }^{8}$ The good agreement of that work with other measurements and theory confirms that our angular distributions are not subject to any appreciable geometric errors. OBP, on the other hand, used a directed beam for a target but treated it as a 
static gas in calculating cross sections. By their own admission ${ }^{1}$ this caused an error which lies someplace between unity and $\sin \theta$, i.e., the correction indicated above with $a$ between unity and zero. We believe that the present data provide a basis for an estimate of the correction needed in the data of OBP.

We have measured DDCS for 100-500-eV electrons impacting upon neon, argon, and nitrogen and $100-\mathrm{eV}$ electrons on hydrogen. Secondary electrons were detected with energies between $4 \mathrm{eV}$ and the primary energy minus the first ionization potential of the target. Angles of emission were $10^{\circ}$ to $150^{\circ}$. The argon data at $500 \mathrm{eV}$ are compared with the recent calculations of Manson. ${ }^{9}$ His calculations, as in the case of helium, were based on the Born approximation with HartreeSlater initial discrete and final continuum wave functions. Only the $3 p$ subshell of argon was considered, but Manson estimate ${ }^{9}$ that the $3 s$ subshell would contribute less than $10 \%$ to the cross sections up to $20 \mathrm{eV}$ and no more than $20 \%$ up to $200 \mathrm{eV}$.

\section{EXPERIMENTAL APPARATUS}

The apparatus was the same as used in I and was described in detail previously. ${ }^{8}$ An electron beam, produced by a rotatable electron gun, is directed. into a static gas target. Secondary electrons emitted at an angle $\theta$ are energy analyzed by a parallel-plate electrostatic analyzer and detected by a channeltron. Measurements of integrated primary beam currents, geometrical factors, detection efficiency, and secondary electron counts detected with and without a target gas present allow absolute values of DDCS to be determined.

DDCS for argon and nitrogen were measured for selected secondary electron energies by manually adjusting the analyzing voltage and recording the scattered signals. For neon and hydrogen the secondary energies were automatically varied in small increments while the scattered signals were reccirded. In both cases the statistical error of the recorded signal with target gas present was less than 5\%. The background correction increased from approximately $5 \%$ for energies greater than $10 \mathrm{eV}$ to $30 \%-60 \%$ for the lowest energies measured. For this reason, the low energy data must be considered to be less reliable. The overall uncertainty in the relative cross sections was generally $\pm 15 \%$ while the absolute values were uncertain by $\pm 20 \%$ for secondary energies above $10 \mathrm{eV}$.

\section{EXPERIMENTAL RESULTS}

Representative graphs of the DDCS for Ne, Ar, $\mathrm{H}_{2}$, and $\mathrm{N}_{2}$ are shown in Figs. 1-4, respectively,

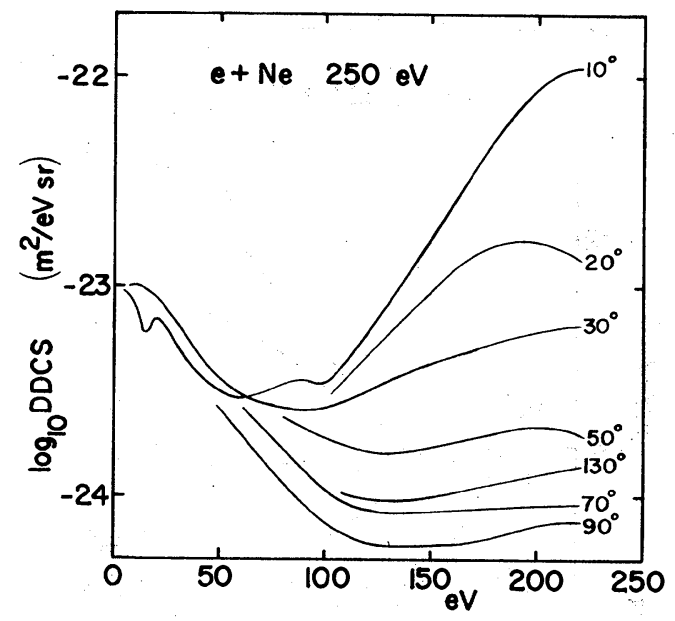

FIG. 1. Doubly differential cross sections for ejection of electrons at various angles vs secondary electron energy for $250-\mathrm{eV} e-\mathrm{Ne}$ collisions.

while Table I provides a numerical listing for some of the cross sections graphed. A complete numerical tabulation of the cross sections for all energies, angles, and gases is available elsewhere. ${ }^{10}$

The DDCS rise at low secondary electron energies for all angles and also at high secondary energies for small angles. Peaks due to Auger transitions are seen in $\mathrm{Ar}$ at $200 \mathrm{eV}$ and in $\mathrm{N}_{2}$ at

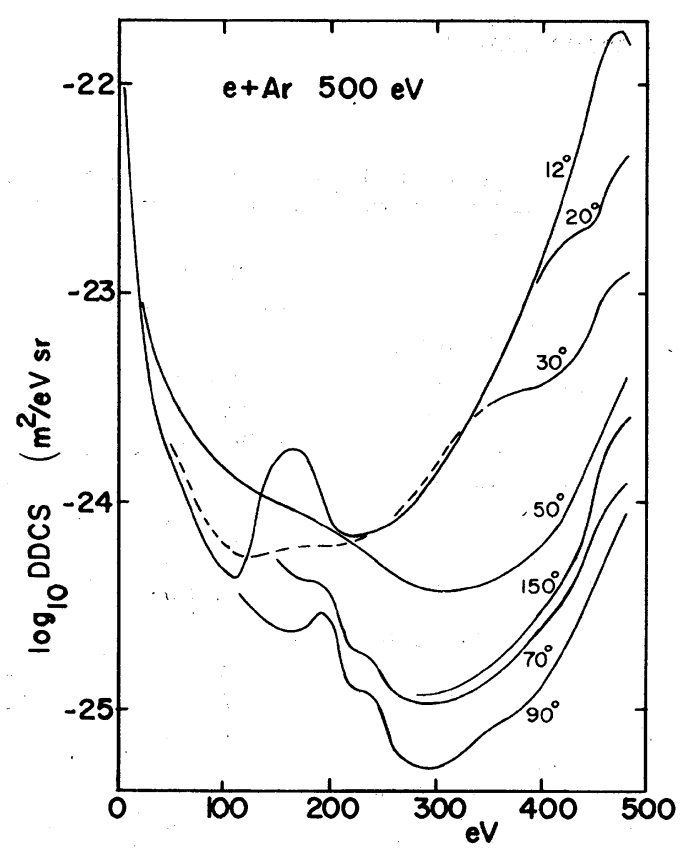

FIG. 2. Doubly differential cross sections vs secondary electron energy for $500-\mathrm{eV} e-\mathrm{Ar}$ collisions. 


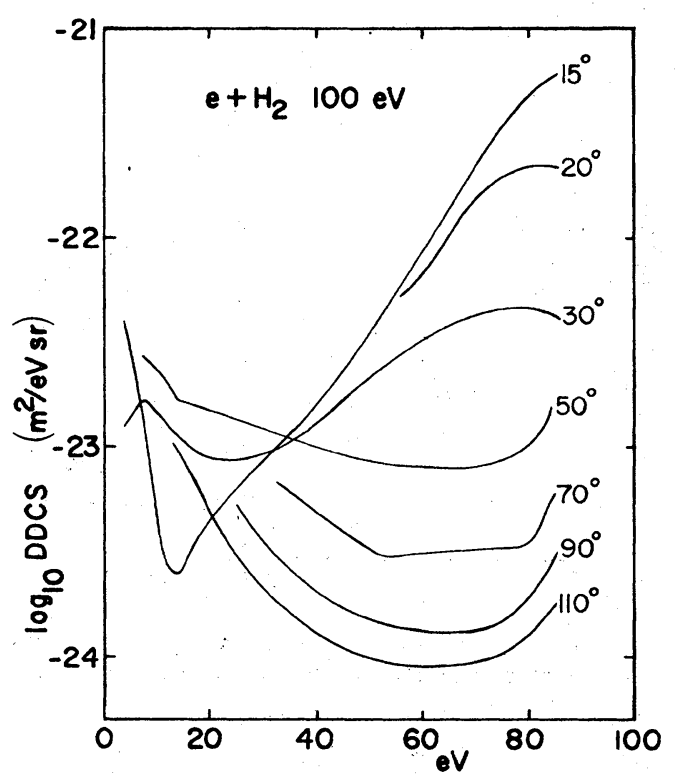

FIG. 3. Doubly differential cross sections vs secondary electron energy for $100-\mathrm{eV} e-\mathrm{H}_{2}$ collisions.

$360 \mathrm{eV}$. The structure observed at an energy loss of approximately $43 \mathrm{eV}$ in neon and $25 \mathrm{eV}$ in argon is probably due to doubly excited states of the target. A spurious structure at 0.32 times the primary energy is attributed to reflections from the analyzer surfaces as it appears in all gases at small angles where a strong elastically scattered electron beam enters the analyzer. The true continuum cross sections should be smooth curves under those bumps.



FIG. 4. Doubly differential cross sections vs secondary electron energy for $500-\mathrm{eV} e-\mathrm{N}_{2}$ collisions.

Figure 5 compares the angular distributions for selected secondary electron energies for the theoretical values of Manson, the measured values of OBP, and the present data for $500-\mathrm{eV}$ argon. As for helium, the measurements of OBP and the present data agree well near $90^{\circ}$ and more poorly at the extreme angles. The theoretical angular distributions appear to agree better with the pre-

TABLE I. Doubly differential cross sections in $\mathrm{m}^{2} / \mathrm{eV}$ sr for selected angles and energies.

\begin{tabular}{cccccc}
\hline \hline & & & & $E_{s} \cdot(\mathrm{eV}) \pm 1 \mathrm{eV}$ \\
0 & 20 & 50 & & 200 & 400 \\
\hline $250-\mathrm{eV} \mathrm{Ne}$ & & & & \\
$30^{\circ}$ & $9.10(-24)$ & $3.67(-24)$ & $2.64(-24)$ & $5.98(-24)$ & \\
$90^{\circ}$ & $6.76(-24)$ & $2.64(-24)$ & $7.64(-25)$ & $7.27(-25)$ & \\
$150^{\circ}$ & $8.29(-24)$ & $3.02(-24)$ & $1.69(-24)$ & $1.98(-24)$ & \\
$500-\mathrm{eV} \mathrm{Ar}$ & & & & & \\
$30^{\circ}$ & $1.21(-23)$ & $1.85(-24)$ & $6.42(-25)$ & $6.24(-25)$ & $3.66(-24)$ \\
$90^{\circ}$ & $1.29(-23)$ & $2.16(-24)$ & $5.51(-25)$ & $2.70(-25)$ & $1.28(-25)$ \\
$150^{\circ}$ & $7.84(-24)$ & $1.44(-24)$ & $5.83(-25)$ & $3.97(-25)$ & $2.92(-25)$ \\
$100-\mathrm{eV} \mathrm{H} \mathrm{H}_{2}$ & & & & & \\
$30^{\circ}$ & $8.84(-24)$ & $2.10(-23)$ & & & \\
$90^{\circ}$ & $6.91(-24)$ & $1.43(-24)$ & & & \\
$145^{\circ}$ & $7.13(-24)$ & $2.08(-24)$ & & & \\
$500-\mathrm{eV} \mathrm{N} \mathrm{N}_{2}$ & & & & & \\
$30^{\circ}$ & $1.65(-23)$ & $2.95(-24)$ & $7.92(-25)$ & $5.21(-25)$ & $4.74(-24)$ \\
$90^{\circ}$ & $1.70(-23)$ & $3.46(-24)$ & $6.65(-25)$ & $1.02(-25)$ & $1.63(-25)$ \\
$150^{\circ}$ & $1.16(-23)$ & $2.24(-24)$ & $4.93(-25)$ & $8.42(-26)$ & $1.08(-25)$ \\
\hline \hline
\end{tabular}


sent measurements than with OBP, particularly for small secondary electron energies; however, the forward peak we observe at small angles and intermediate secondary energies is absent from the calculations. As was pointed out previously, ${ }^{11}$ the forward peak is attributed to electron exchange. Hence its absence in the calculations, which do not include exchange, is not surprising. It should be noted that recently Oda and Nishimura $^{12}$ presented improved measurements which did not show the rise in the forward direction formerly reported by them and seen in the present work.

In Fig. 6 we have plotted the ratio of OBP's measurements to the theoretical values of Manson for $500-\mathrm{eV}$ argon. The ratios are normalized at $90^{\circ}$ and averaged over secondary energies between 0 and $30 \mathrm{eV}$, where the calculations should be most accurate. Also shown is a similar comparison of the OBP data with our present measurements but

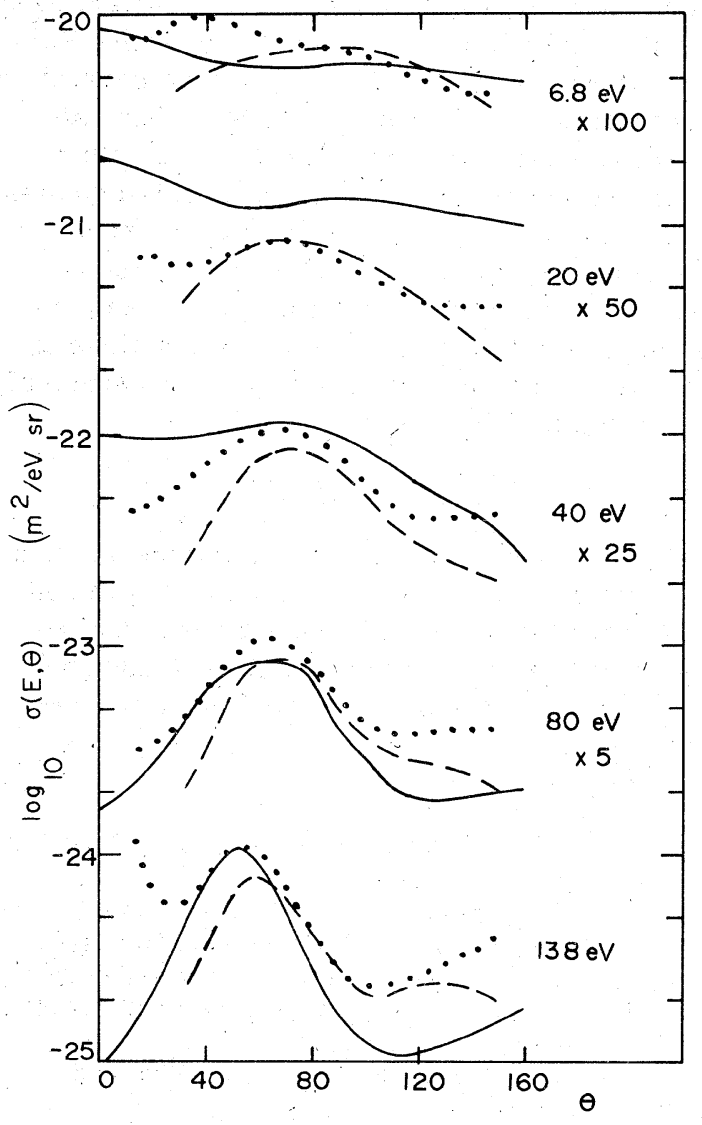

FIG. 5. Doubly differential cross sections for ejection of electrons at various energies vs angle of emission for $500-\mathrm{eV}$ electrons on argon. Solid curve: theory by Manson (Ref. 9); dotted curve: present data; dashed curve: data of Opal, Beaty, and Peterson (Ref. 1).

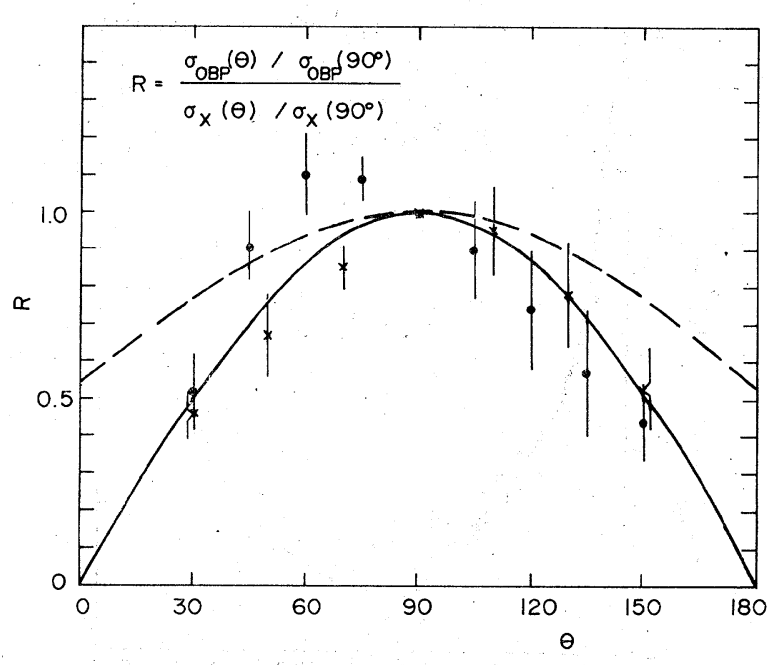

FIG. 6. Ratios of OBP data to theory by Manson (๑) and to present data $(x)$ for $500-\mathrm{eV}$ argon. Ratios are normalized to $90^{\circ}$. Solid curve is a least-squares fit to $\mathrm{OBP} /$ Manson ratios of form $a+(1-a) \sin \theta$, with $a=0.02 \pm 0.14$. Dashed curve with $a=0.53$ is suggested correction to OBP data obtained from helium data analysis (Ref. 6).

in this case averaged over a range of energies between 20 and $200 \mathrm{eV}$, where the measurements should be most accurate. To obtain the ratios at $50^{\circ}, 70^{\circ}, 110^{\circ}$, and $130^{\circ}$ a logarithmic interpolation was applied to the OBP data. The solid curve of the form $a+(1-a) \sin \theta$ is a least-squares fit to the OBP/Manson ratios with $a=0.02 \pm 0.14$. A similar fit for the OBP/present ratios yields $a=-0.05 \pm 0.06$. Also shown by the dashed curve is the correction with $a=0.53$ which we suggested earlier for the helium data.

Extending the comparison between OBP and the present data to other gases and energies gives values of $a$ ranging from -0.05 to 0.21 . Averaging all of the present experimental data gives a value of $a=0.10 \pm 0.12$.

The measurements of Tisone ${ }^{5}$ for $500-\mathrm{eV}$ electrons on $\mathrm{N}_{2}$ agree well with the present data. The angular distributions for the various electron energies generally agrees within $10 \%$.

For secondary electron energies above $10 \mathrm{eV}$ our DDCS at $90^{\circ}$ agree well with those of OBP, the discrepancies being generally $4 \%-8 \%$. However, for neon at $500 \mathrm{eV}$ our data were $20 \%$ larger at $200 \mathrm{eV}$ and about $30 \%$ smaller at $10 \mathrm{eV}$.

The DDCS may be integrated over angle to obtain the singly differential cross sections (SDCS) by the relation

$$
\sigma\left(E_{s}\right)=2 \pi \int_{0}^{\pi} \sigma\left(E_{s}, \theta\right) \sin \theta d \theta
$$




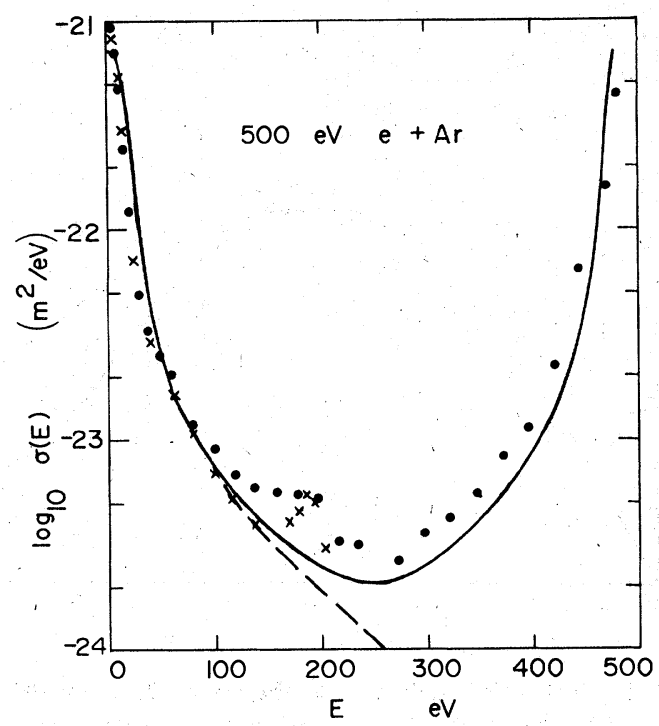

FIG. 7. Singly differential cross sections vs secondary electron energy for 500-eV argon: $\bullet$, present data; $\times$, data by Opal, Beaty, and Peterson (Ref. 1); dashed curve, theory by Manson (Ref. 9), ejected electrons only; solid curve, theory by Manson, ejected plus scattered electrons.

Cross sections differential only in angle $\theta$ were also obtained by integration over secondary energy. Thus

$$
\sigma(\theta)=\int_{0}^{E_{p}-I} \sigma\left(E_{s}, \theta\right) d E_{s} .
$$

These integrations were done using the trapezoidal rule, i.e., a linear interpolation between data points. Figure 7 gives the singly differential cross sections $\sigma\left(E_{s}\right)$ for $500-\mathrm{eV}$ argon. The calculations of Manson have been "folded" to include both scattered and ejected electron contributions. The agreement is rather good over the entire ionization continuum, although the calculated energy dependence slightly underestimates the contribution of intermediate energy electrons probably because exchange was not included. Also shown are the OBP data, which agree quite well with the present cross sections. $\mathrm{Kim}^{13}$ has previously demonstrated that the SDCS of OBP are quite good at low energies. The increased disagreement for the larger energies is probably due to their underestimation of the DDCS at $30^{\circ}$ and its effect on the integration over angles. For larger energies the contribution of the small angles to the SDCS increases.

\section{CONCLUSIONS}

We have presented the angular and energy dependence of the absolute doubly differential cross sections for secondary electron emission from neon, argon, hydrogen, and nitrogen. The present data agree well with measurements of OBP at $90^{\circ}$ but suggest that their angular distributions would be improved if their data were divided by the factor $0.10+0.90 \sin \theta$. This correction is confirmed by the measurements of Tisone on $\mathrm{N}_{2}$. Thus the experimental data indicate a need for a correction of OBP's data which is very near $\sin \theta$ while Manson's theoretical calculations require a correction between $\sin \theta$ and $\frac{1}{2}(1+\sin \theta)$. While we have attempted to fit the angular discrepancies with a simple mathematical expression, Fig. 6 indicates that a more exact correction would require a more complicated form. Better calculations or more definitive measurements may be required to ascertain the precise angular distributions of secondary electrons and to verify the presence of the forward peak seen here.

\section{ACKNOWLEDGMENTS}

The authors wish to thank S. T. Manson for providing his unpublished calculations on argon and W. K. Peterson for useful suggestions and discussion. We also wish to thank E. P. Rudd and R. Morris for assisting with the computer work.
*Present address: Institute of Physics, University of Aarhus, DK 8000 Aarhus C, Denmark.

${ }^{1}$ C. B. Opal, E. C. Beaty, and W. K. Peterson, At. Data 4, 209 (1972); W. K. Peterson, C. B. Opal, and E. C. Beaty, J. Phys. B 4, $1020 \cdot(1971)$; W. K. Peterson, E. C. Beaty, and C. B. Opal, Phys. Rev. A 5, 712 (1972); C. B. Opal, W. K. Peterson, and E. C. Beaty, J. Chem. Phys. 55, 4100 (1971); W. K. Peterson, thesis (University of Colorado, 1971) (unpublished); E. C. Beaty, Radiat. Res. 64, 70 (1975).

${ }^{2}$ N. Oda, Radiat. Res. $\overline{64}, 80$ (1975).

${ }^{3}$ R. E. Kennerly and R. A. Bonham, in Abstracts of the Fifth International Conference on Atomic Physics, Berkeley, 1976, edited by R. Marrus, M. H. Prior, and H. A . Shugart (University of California, Berkeley, 1976), p. 88.

${ }^{4} \mathrm{~L}$. H. Toburen and W. C. Wilson, in Abstracts of Papers of the Eighth International Conference on the Physics of Electronic and Atomic Collisions, Belgrade, 1973, edited by B. C. Čobić and M. V. Kurepa (Institute of Physics, Belgrade, 1973), p. 693.

${ }^{5}$ E. C. Tisone, J. Chem. Phys. 56, 108 (1972); and private communication.

${ }^{6}$ M. E. Rudd and R. D. DuBois, Phys. Rev. A 16, 26 (1977).

${ }^{7}$ S. T. Manson, L. H. Toburen, D. H. Madison, and N. Stolterfoht, Phys. Rev. A 12, 60 (1975).

${ }^{8}$ R. D. DuBois and M. E. Rudd, J. Phys. B 8,1474 
(1975); 9, 2657 (1976)

${ }^{9}$ S. T. Manson (private communication).

${ }^{10}$ See AIP document No. PAPS PLRAA-17-843-24 for

24 pages of tables of cross sections. Order by PAPS number and journal reference from American Institute of Physics Auxiliary Publication Service, 335 East 45th Street, New York, N.Y. 10017. The price is $\$ 1.50$ for each microfiche (98 pages), or $\$ 5$ for photocopies of up to 30 pages with $\$ 0.15$ for each additional page over 30 pages. Airmail additional. Make checks payable to the American Institute of Physics. This material also appears in Current Physics Microfilm, the monthly microfilm edition of the complete set of journals published by AIP, on the frames immediately following this journal article.

${ }^{11} \mathrm{~S}$. Tahira and N. Oda, J. Phys. Soc. Jpn. 35, 582 (1973).

${ }^{12} \mathrm{~N}$. Oda and F. Nishimura, in Abstracts of Papers of the Tenth International Conference on the Physics of Electronic and Atomic Collisions, Paris, 1977, edited by M. Barat and J. Reinhardt (Commissariat a l'Energie A tomique, Paris, 1977), p. 362.

${ }^{13}$ Y. K. Kim and M. Inokuti, Phys. Rev. A 7, 1257 (1973); Y. K. Kim, Radiat. Res. 61, 21 (1975); 64, 96 (1975); 64, 205 (1975); Y. K. Kim and T. Noguchi, Int. J. Radiat. Phys. Chem. 7 , 77 (1975). 